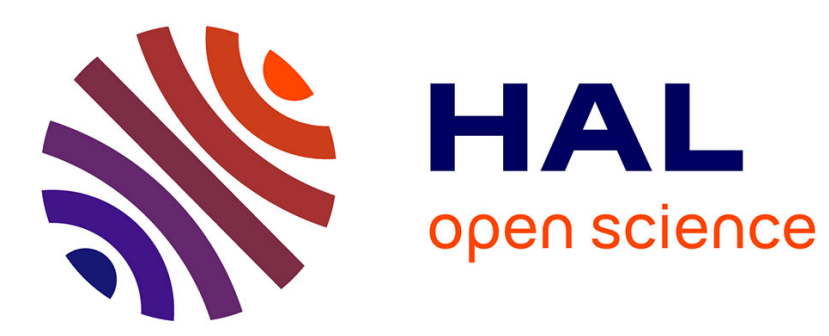

\title{
Reachability and confluence are undecidable for flat term rewriting systems
}

Florent Jacquemard

\section{To cite this version:}

Florent Jacquemard. Reachability and confluence are undecidable for flat term rewriting systems. Information Processing Letters, 2003, 87 (5), pp.265-270. inria-00578875

\section{HAL Id: inria-00578875 \\ https://hal.inria.fr/inria-00578875}

Submitted on 22 Mar 2011

HAL is a multi-disciplinary open access archive for the deposit and dissemination of scientific research documents, whether they are published or not. The documents may come from teaching and research institutions in France or abroad, or from public or private research centers.
L'archive ouverte pluridisciplinaire HAL, est destinée au dépôt et à la diffusion de documents scientifiques de niveau recherche, publiés ou non, émanant des établissements d'enseignement et de recherche français ou étrangers, des laboratoires publics ou privés. 


\title{
Reachability and confluence are undecidable for flat term rewriting systems
}

\author{
Florent Jacquemard \\ INRIA Futurs \& LSV/CNRS UMR 8643, ENS de Cachan, \\ 61, avenue du président-Wilson \\ 94235 Cachan Cedex, France \\ Phone: +33-1 47407544 Fax: +33-1 47402464 \\ florent. jacquemard@lsv. ens-cachan.fr
}

\begin{abstract}
Ground reachability, ground joinability and confluence are shown undecidable for flat term rewriting systems, i.e. systems in which all left and right members of rule have depth at most one.
\end{abstract}

\section{Introduction}

The confluence of a term rewriting systems (TRS) guarantees that every term has at most one normal form. This property is undecidable in general, and has been shown decidable for ground TRS in $[1,2]$. The main result of [1] also implies the decidability of the reachability and joinability problems for ground TRS.

More recently, the confluence has been shown solvable in polynomial time for several classes of TRS, every class embedding the previous one: some restricted ground TRS in [3], ground TRS in [4], shallow (variables occur at depth at most 1 in rewrite rules) and rule linear (in every rewrite rule, every variable occurs at most once) TRS in [5], and shallow, linear (in every left or right member of rewrite rule, every variable occurs at most once) TRS in [6]. The polynomial time complexity result of [4] is also valid for the decision of reachability and joinability, which was already shown in [1].

Reachability, joinability and confluence are undecidable for linear (nonshallow) TRS [7], but it was not known whether we can relax the linearity assumptions on variables of the systems of $[5,6]$, keeping these properties decidable $^{1}$. We answer here by the negative, showing, with a reduction of the Post Correspondence Problem, that the problems of ground reachability, ground joinability and confluence are undecidable for flat TRS (every terms in rewrite rules have depth at most 1) with non linear variables. The proof

\footnotetext{
${ }^{1}$ Reachability, joinability and confluence are shown NP-hard in [6].
} 
for ground reachability uses the same colored techniques as an older proof of undecidability of rigid reachability [8], though this latter result could not be reused directly in this context.

\section{Preliminaries}

Given a signature $\Sigma$, and a set of variable symbols $\mathcal{X}$, we note $\mathcal{T}(\Sigma, \mathcal{X})$ the set of terms build with symbols of $\Sigma$ and $\mathcal{X}$ and $\mathcal{T}(\Sigma)$ its subset of ground terms. The set of function symbols of $\Sigma$ of arity $i$ is denoted $\Sigma_{i}$.

A term rewriting system (TRS) on $\Sigma$ is defined as a finite set of rewrite rules denoted $\ell \rightarrow r$ with $\ell, r \in \mathcal{T}(\Sigma, \mathcal{X})$. We note $\rightarrow R$ the rewrite relation (on terms of $\mathcal{T}(\Sigma, \mathcal{X})$ ) defined by the TRS $R$, and $\underset{R}{*}$ the reflexive and transitive of this relation.

Definition 1 A TRS $R$ is called shallow (respectively flat), if all its rewrite rules have the form $f\left(t_{1}, \ldots, t_{n}\right) \rightarrow g\left(s_{1}, \ldots, s_{m}\right)$ or $x \rightarrow g\left(s_{1}, \ldots, s_{m}\right)$ or $f\left(t_{1}, \ldots, t_{n}\right) \rightarrow x$ where every $t_{i}$ and $s_{i}$ is either a variable of $\mathcal{X}$ or a ground term of $\mathcal{T}(\Sigma)$ (respectively a variable of $\mathcal{X}$ or a symbol of $\Sigma_{0}$ ), and where $x \in \mathcal{X}$, and $n, m$ can be 0 (if $f$ or $g$ have arity 0 ).

We are interested in the following decision problems:

(ground) reachability. Given a TRS $R$ on a signature $\Sigma$ and two (ground) terms $s, t \in \mathcal{T}(\Sigma, \mathcal{X})$, do we have a reduction $s \stackrel{*}{R} t$ ?

(ground) joinability. Given a TRS $R$ on $\Sigma$ and two (ground) terms $s, t \in \mathcal{T}(\Sigma, \mathcal{X})$, does there exists $v \in \mathcal{T}(\Sigma, \mathcal{X})$ such that $s \stackrel{*}{R} v \stackrel{*}{R} t$ ?

confluence. Given a TRS $R$ on $\Sigma$, do we have: for all $s, t \in \mathcal{T}(\Sigma, \mathcal{X})$ such that $s \stackrel{*}{R} u \stackrel{*}{R} t$ for some $u \in \mathcal{T}(\Sigma, \mathcal{X})$, does there exists $v \in \mathcal{T}(\Sigma, \mathcal{X})$ such that $s \stackrel{*}{R} v \stackrel{*}{R} t$ ?

We shall show below that the ground reachability, ground joinability and confluence problems are undecidable for flat TRS, by reduction of the Post correspondence problem.

\section{Post Correspondence Problem, coding and col- oring}

We consider an instance of the Post Correspondence Problem (PCP) given by a finite set of pairs of words:

$$
\mathrm{PCP}:=\left\{\left(u_{i}, v_{i}\right) \mid u_{i}, v_{i} \in\{a, b\}^{*}, 1 \leq i \leq N\right\}
$$

The following problem is undecidable: 
Does there exist a finite sequence $\left(i_{j}\right)_{0 \leq j \leq k}$ with $1 \leq i_{0}, \ldots, i_{k} \leq$ $N$, such that $u_{i_{0}} u_{i_{1}} \ldots u_{i_{k}}=v_{i_{0}} v_{i_{1}} \ldots v_{i_{k}}$ ?

We shall represent the hypothetical solutions of PCP by ground terms from the sets described in Section 2.1, and provide in Sections 3, 4, and 5 some reductions to the reachability, joinability and confluence decision. The ingredients for the construction of the TRSs used in the reductions are two automata (Section 2.2), four TRSs (beginning of Section 3) and some coloring (Section 2.3).

\subsection{Product and string terms}

Let - be a new symbol. We shall use a product operator $\otimes$ which associate to two words of $\{a, b\}^{*}$ a word of $\{a, b,\}^{2^{*}}$ as follows:

$$
c_{1} \ldots c_{n} \otimes c_{1}^{\prime} \ldots c_{m}^{\prime}:=\left\langle c_{1}, c_{1}^{\prime}\right\rangle \ldots\left\langle c_{k}, c_{k}^{\prime}\right\rangle
$$

where $c_{1}, \ldots, c_{n}, c_{1}^{\prime}, \ldots, c_{m}^{\prime} \in\{a, b\}, k=\max (n, m)$, and for all $i$ with $n<$ $i \leq k$, if any, (resp. all $j$ with $m<j \leq k), c_{i}=$ - (resp. $\left.c_{j}^{\prime}={ }_{-}\right)$.

Example $2 a \otimes b a b=\langle a, b\rangle\left\langle_{-}, a\right\rangle\left\langle_{-}, b\right\rangle$.

Let us consider the signature $\Gamma:=\{a, b, \varepsilon\}$, where $a, b$ and $\varepsilon$ have the respective arities 1,1 and 0 .

We write: $\Gamma_{-}:=\Gamma \uplus\left\{\left\{_{-}\right\}\right.$, where $\_$has arity 0 in $\Gamma_{-}$, and $\Delta:=\left\{a, b,{ }_{-}\right\}^{2} \cup\{\varepsilon\}$, where $\varepsilon$ has arity 0 in $\Delta$, and every other symbols have arity 1 in $\Delta$.

Remark 3 We make no distinctions below between a word $c_{1} \ldots c_{n} \in\{a, b\}^{*}$ (resp. $d_{1} \ldots d_{n} \in\left\{a, b,{ }_{-}\right\}^{2^{*}}$ ) and the ground term $c_{1}\left(\ldots c_{n}(\varepsilon)\right) \in \mathcal{T}(\Gamma)$ (resp. $\left.d_{1}\left(\ldots d_{n}(\varepsilon)\right) \in \mathcal{T}(\Delta)\right)$.

In this manner, the operator $\otimes$ is extended to $\mathcal{T}(\Gamma) \times \mathcal{T}(\Gamma) \rightarrow \mathcal{T}(\Delta)$.

\subsection{Automata associated to PCP}

Let $A$ and $B$ be two finite automata recognizing the respective sets: $L(A)=$ $\left\{u_{i} \otimes v_{i} \mid 1 \leq i \leq N\right\}^{*}$ and $L(B)=\{a, b\}^{*}$, and with respective state sets $Q_{A}$ and $Q_{B}$ and initial states $q_{A}$ and $q_{B}$. Following Remark 3 , we shall consider $L(A)$ and $L(B)$ as subsets of, respectively, $\mathcal{T}(\Delta)$ and $\mathcal{T}(\Gamma)$.

We associate to $A$ and $B$ two ground TRS $T_{A}$ and $T_{B}$ on the respective signatures $\Delta \uplus Q_{A}$ and $\Gamma \uplus Q_{B}$, where the states symbols of $Q_{A}$ and $Q_{B}$ have arity 0 , as follows:

$$
\begin{aligned}
T_{A}:= & \left\{q \rightarrow d\left(q^{\prime}\right) \mid q, q^{\prime} \in Q_{A}, d \in \Delta, q \stackrel{d}{\rightarrow} q^{\prime} \text { is a transition of } A\right\} \\
& \cup\left\{q \rightarrow q^{\prime} \mid q, q^{\prime} \in Q_{A}, q \rightarrow q^{\prime} \text { is an epsilon-transition of } A\right\} \\
& \cup\left\{q \rightarrow \varepsilon \mid q \in Q_{A} \text { is a final state of } A\right\}
\end{aligned}
$$




$$
\begin{aligned}
T_{B}:= & \left\{q \rightarrow c\left(q^{\prime}\right) \mid q, q^{\prime} \in Q_{B}, c \in \Gamma, q \stackrel{c}{\rightarrow} q^{\prime} \text { is a transition of } B\right\} \\
& \cup\left\{q \rightarrow q^{\prime} \mid q, q^{\prime} \in Q_{B}, q \rightarrow q^{\prime} \text { is an epsilon-transition of } B\right\} \\
& \cup\left\{q \rightarrow \varepsilon \mid q \in Q_{B} \text { is a final state of } B\right\}
\end{aligned}
$$

\subsection{Coloring terms and TRS}

We assume given 19 disjoint copies of the above signatures, colored with color $i$ for $0 \leq i \leq 18: \Gamma_{-}^{(i)}:=\left\{c^{(i)} \mid c \in \Gamma_{-}\right\}, Q_{A}^{(i)}:=\left\{q^{(i)} \mid q \in Q_{A}\right\}$, $Q_{B}^{(i)}:=\left\{q^{(i)} \mid q \in Q_{B}\right\}, \Delta^{(i)}:=\left\{\left\langle c^{(i)}, c^{(i)}\right\rangle \mid\left\langle c, c^{\prime}\right\rangle \in \Delta\right\}$.

Let $\Theta$ be the following signature $\Theta:=\Gamma_{-} \cup \Delta \cup Q_{A} \cup Q_{B}$, where the symbols of $\Gamma_{-}$and $\Delta$ keep their respective arities in $\Theta$ and the symbols of $Q_{A}$ and $Q_{B}$ have arity 0 in $\Theta$, and let and $\Theta^{(i)}(0 \leq i \leq 18)$ be the colored copies of $\Theta, \Theta^{(i)}:=\Gamma_{-}{ }^{(i)} \cup \Delta^{(i)} \cup Q_{A}^{(i)} \cup Q_{B}^{(i)}$.

For $0 \leq i \leq 18$, the $i$-coloring $t^{(i)} \in \mathcal{T}\left(\Theta^{(i)}, \mathcal{X}\right)$ of a term of $t \in \mathcal{T}(\Theta, \mathcal{X})$ is recursively defined by: $f(t)^{(i)}:=f^{(i)}\left(t^{(i)}\right)$, and $x^{(i)}:=x$ for all $x \in \mathcal{X}$.

Given a set $U \subseteq \mathcal{T}(\Theta)$, we write $U^{(i)}:=\left\{t^{(i)} \mid t \in U\right\}$, and given a TRS $R$ on $\Theta$, we let $R^{(i, j)}:=\left\{l^{(i)} \rightarrow r^{(j)} \mid l \rightarrow r \in R\right\}$ and $R^{(i)}:=R^{(i, i)}$.

\section{Reduction of PCP to reachability for flat TRS}

We associate a TRS $R_{1}$ to the above problem PCP in (8), see also Figure 1. Its definition refers to the following two trivial and two projections TRS:

$$
\begin{aligned}
S:= & \left\{c(x) \rightarrow c(x) \mid c \in \Gamma_{1}\right\} \cup\{\varepsilon \rightarrow \varepsilon\} \\
P:= & \left\{d(x) \rightarrow d(x) \mid d \in \Delta_{1}\right\} \cup\{\varepsilon \rightarrow \varepsilon\} \\
\Pi_{1}:= & \left\{\left\langle c, c^{\prime}\right\rangle(x) \rightarrow c(x) \mid c \in \Gamma_{1}, c^{\prime} \in \Gamma_{-}\right\} \\
& \cup\left\{\left\langle{ }_{-}, c^{\prime}\right\rangle(x) \rightarrow x \mid c^{\prime} \in \Gamma_{1}\right\} \cup\{\varepsilon \rightarrow \varepsilon\} \\
\Pi_{2}:= & \left\{\left\langle c, c^{\prime}\right\rangle(x) \rightarrow c^{\prime}(x) \mid c \in \Gamma_{-}, c^{\prime} \in \Gamma_{1}\right\} \\
& \cup\left\{\left\langle c,,_{-}\right\rangle(x) \rightarrow x \mid c \in \Gamma_{1}\right\} \cup \cup\{\varepsilon \rightarrow \varepsilon\}
\end{aligned}
$$

The identity TRS $S$ and $P$ shall of course be used only in their colored form $S^{(i, j)}$ and $P^{(i, j)}$.

Example 4 Let $u_{1}=a, v_{1}=b a b, u_{2}=a b, v_{2}=b$.

$$
\begin{aligned}
& \left(u_{1} \otimes v_{1}\right)\left(u_{2} \otimes v_{2}\right)=\langle a, b\rangle\left(\left\langle_{-}, a\right\rangle\left(\left\langle_{-}, b\right\rangle\left(\langle a, b\rangle\left(\left\langle b,,_{-}\right\rangle(\varepsilon)\right)\right)\right)\right) \underset{\Pi_{1}}{\longrightarrow} \\
& a\left(\left\langle_{-}, a\right\rangle\left(\left\langle_{-}, b\right\rangle\left(\langle a, b\rangle\left(\left\langle b,{ }_{-}\right\rangle(\varepsilon)\right)\right)\right)\right) \underset{\Pi_{1}}{\longrightarrow} a\left(\left\langle_{-}, b\right\rangle\left(\langle a, b\rangle\left(\left\langle b,,_{-}\right\rangle(\varepsilon)\right)\right)\right) \underset{\Pi_{1}}{\longrightarrow} \\
& a\left(\langle a, b\rangle\left(\left\langle b,,_{-}\right\rangle(\varepsilon)\right)\right) \underset{\Pi_{1}}{\longrightarrow} a\left(a\left(\left\langle b,,_{-}\right\rangle(\varepsilon)\right)\right) \underset{\Pi_{1}}{\longrightarrow} a(a(b(\varepsilon)))
\end{aligned}
$$

The TRS $R_{1}$ is defined on an extended signature: $\Xi=\bigcup_{i=0}^{i=18} \Theta^{(i)} \uplus$ $\{f, g, 0,1\}$ where $f, g, 0,1$ are new function symbols of respective arities 8 , $8,0,0$ in $\Xi$. 


$$
\begin{aligned}
& 0 \rightarrow f\left(q_{A}^{(3)}, \quad q_{A}^{(4)}, \quad q_{A}^{(5)}, \quad q_{B}^{(13)}, \quad q_{B}^{(14)}, \quad q_{A}^{(6)}, \quad q_{B}^{(15)}, \quad q_{B}^{(16)}\right) \\
& T_{A}^{(3)} \quad T_{A}^{(4)} \quad T_{A}^{(5)} \quad T_{B}^{(13)} \quad T_{B}^{(14)} \quad T_{A}^{(6)} \quad T_{B}^{(15)} \quad T_{B}^{(16)} \\
& P^{(3,1)} \quad P^{(4,2)} \quad P^{(5,1)} \quad S^{(13,11)} \quad S^{(14,12)} \quad P^{(6,2)} \quad S^{(15,11)} \quad S^{(16,12)} \\
& f\left(x_{1}, \quad x_{2}, \quad x_{1}, \quad y_{11}, \quad y_{12}, \quad x_{2}, \quad y_{11}, \quad y_{12}\right) \\
& g\left(x_{1}, \quad x_{2}, \quad x_{1}, \quad y_{11}, \quad y_{12}, \quad x_{2}, \quad y_{11}, \quad y_{12}\right) \\
& P^{(1,0)} \quad P^{(2,0)} \quad \Pi_{1}^{(1,17)} \quad S^{(11,17)} \quad S^{(12,18)} \quad \Pi_{2}^{(2,18)} \quad S^{(11,10)} \quad S^{(12,10)}
\end{aligned}
$$

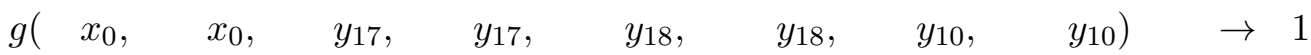

Figure 1: The TRS $R_{1}$. The placement of the rules illustrates the equivalence between the existence of a solution to $\mathrm{PCP}$ and the existence of a reduction $0 \underset{R_{1}}{\stackrel{*}{\longrightarrow}}$. A solution is represented by a term $s \in L(A)$ such that $s \underset{\Pi_{1}}{\stackrel{*}{\longrightarrow}}$ $t \stackrel{*}{\Pi_{2}} s$ for some $t \in L(B)$. The terms $s$ and $t$ are duplicated in the reduction (with different colors), they correspond to the variables $x_{i}$ and $y_{j}$ respectively. In the reduction, the rules of the top part (above $f(\ldots) \rightarrow$ $g(\ldots))$ ensures that the (instances of) $x_{i}$ and $y_{j}$ belong respectively to $L(A)$ and $L(B)$ and the rules of the bottom part ensure the above relation between $s$ and $t$, namely $x_{1}$ and $x_{2}$ are the same term $x_{0}$, modulo coloring, the projection with $\Pi_{1}$ of $x_{1}$ is $y_{17}$, the projection with $\Pi_{2}$ of $x_{2}$ is $y_{18}$, and $y_{17}$ and $y_{18}$ are the same term $y_{10}$ modulo coloring.

$$
\begin{aligned}
R_{1}:= & R_{0} \cup\left\{\begin{array}{l}
0 \rightarrow f\left(q_{A}^{(3)}, q_{A}^{(4)}, q_{A}^{(5)}, q_{B}^{(13)}, q_{B}^{(14)}, q_{A}^{(6)}, q_{B}^{(15)}, q_{B}^{(16)}\right), \\
f\left(x_{1}, x_{2}, x_{1}, y_{11}, y_{12}, x_{2}, y_{11}, y_{12}\right) \rightarrow g\left(x_{1}, x_{2}, x_{1}, y_{11}, y_{12}, x_{2}, y_{11}, y_{12}\right), \\
g\left(x_{0}, x_{0}, y_{17}, y_{17}, y_{18}, y_{18}, y_{10}, y_{10}\right) \rightarrow 1
\end{array}\right\} \\
R_{0}:= & T_{A}^{(3)} \cup T_{A}^{(4)} \cup T_{A}^{(5)} \cup T_{A}^{(6)} \cup T_{B}^{(13)} \cup T_{B}^{(14)} \cup T_{B}^{(15)} \cup T_{B}^{(16)} \cup \\
& P^{(3,1)} \cup P^{(4,2)} \cup P^{(5,1)} \cup S^{(13,11)} \cup S^{(14,12)} \cup P^{(6,2)} \cup S^{(15,11)} \cup S^{(16,12)} \cup \\
& P^{(1,0)} \cup P^{(2,0)} \cup \Pi_{1}^{(1,17)} \cup S^{(11,17)} \cup S^{(12,18)} \cup \Pi_{2}^{(2,18)} \cup S^{(11,10)} \cup S^{(12,10)}
\end{aligned}
$$

Note that $R_{1}$ is a flat TRS.

Definition $5 A$ 01-derivation witness for $R_{1}$ is a tuple $\left(s_{0}, s_{1}, s_{2}, t_{10}, t_{11}, t_{12}, t_{17}, t_{18}\right)$ of terms of $\mathcal{T}(\Xi, \mathcal{X})$ such that: $f\left(q_{A}^{(3)}, q_{A}^{(4)}, q_{A}^{(5)}, q_{B}^{(13)}, q_{B}^{(14)}, q_{A}^{(6)}, q_{B}^{(15)}, q_{B}^{(16)}\right)$ $\underset{R_{1}}{\stackrel{*}{\longrightarrow}} f\left(s_{1}, s_{2}, s_{1}, t_{11}, t_{12}, s_{2}, t_{11}, t_{12}\right) \underset{R_{1}}{\longrightarrow} g\left(s_{1}, s_{2}, s_{1}, t_{11}, t_{12}, s_{2}, t_{11}, t_{12}\right) \underset{R_{1}}{\longrightarrow}$ $g\left(s_{0}, s_{0}, t_{17}, t_{17}, t_{18}, t_{18}, t_{10}, t_{10}\right)$.

Lemma $6 \quad 0 \stackrel{*}{\underset{R_{1}}{\longrightarrow}} 1$ iff there exists a 01-derivation witness for $R_{1}$. 
Lemma 7 Every 01-derivation witness for $R_{1} w=\left(s_{0}, s_{1}, s_{2}, t_{10}, t_{11}, t_{12}, t_{17}, t_{18}\right)$ is such that:

$$
\begin{aligned}
& \text { 1. } s_{0} \in L(A)^{(0)}, s_{1} \in L(A)^{(1)}, s_{2} \in L(A)^{(2)}, t_{10} \in L(B)^{(10)}, t_{11} \in \\
& L(B)^{(11)}, t_{12} \in L(B)^{(12)}, t_{17} \in L(B)^{(17)} \text { and } t_{18} \in L(B)^{(18)}, \\
& \text { 2. } s_{1} \frac{*}{P^{(1,0)}} s_{0} \frac{*}{P^{(2,0)}} s_{2} \text {, and } t_{11} \frac{*}{S^{(11,10)}} t_{10} \underset{S^{(12,10)}}{\frac{*}{12}}, \\
& \text { 3. } s_{1} \frac{*}{\Pi_{1}^{(1,17)}} t_{17} \frac{*}{S^{(11,17)}} t_{11} \text {, and } t_{12} \frac{*}{S^{(12,18)}} t_{18} \stackrel{*}{\Pi_{2}^{(2,18)}} s_{2} .
\end{aligned}
$$

Proof: An analysis on the occurrences of symbols in the rules of $R_{1}$ shows that the reductions in Definition 5 contain on one hand (see also Figure 1): $q_{A}^{(3)} \underset{R_{0}}{\stackrel{*}{\longrightarrow}} s_{1}, q_{A}^{(4)} \underset{R_{0}}{\stackrel{*}{\longrightarrow}} s_{2}, q_{A}^{(5)} \underset{R_{0}}{\stackrel{*}{\longrightarrow}} s_{1}, q_{B}^{(13)} \underset{R_{0}}{\stackrel{*}{\longrightarrow}} t_{11}, q_{B}^{(14)} \underset{R_{0}}{\stackrel{*}{R_{12}}} t_{12}$, $q_{A}^{(6)} \underset{R_{0}}{\stackrel{*}{R_{2}}} s_{2}, q_{B}^{(15)} \underset{R_{0}}{\stackrel{*}{R_{11}}} t_{11}, q_{B}^{(16)} \underset{R_{0}}{\stackrel{*}{\longrightarrow}} t_{12}$, and on the other hand: $s_{1} \underset{R_{0}}{\stackrel{*}{\longrightarrow}} s_{0} \stackrel{*}{R_{0}} s_{2}$, $s_{1} \stackrel{*}{R_{0}} t_{17} \stackrel{*}{R_{0}} t_{11}, t_{12} \underset{R_{0}}{\stackrel{*}{\rightarrow}} t_{18} \stackrel{*}{R_{0}} s_{2}, t_{11} \underset{R_{0}}{\stackrel{*}{\longrightarrow}} t_{10} \stackrel{*}{R_{0}} t_{12}$.

The use of colors in the construction of $R_{0}$ implies (1) for $w$. For instance, in $q_{A}^{(3)} \underset{R_{0}}{\stackrel{*}{R_{1}}} s_{1} \stackrel{*}{R_{0}} q_{A}^{(5)}$, because of the coloring with colors 3 and 5 , the left derivation can only involve rules of the sub systems $T_{A}^{(3)}, P^{(3,1)}, P^{(1,0)}$, $\Pi_{1}^{(1,17)}$, and the right derivation can only involve rules of the sub systems $T_{A}^{(5)}, P^{(5,1)}, P^{(1,0)}, \Pi_{1}^{(1,17)}$. Hence, $s_{1} \in \mathcal{T}\left(\Delta^{(0)} \cup \Delta^{(1)} \cup \Gamma^{(17)}\right)$. Similarly,

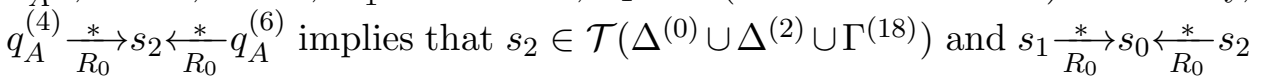
implies that $s_{0} \in \mathcal{T}\left(\Delta^{(0)}\right), s_{1} \in \mathcal{T}\left(\Delta^{(0)} \cup \Delta^{(1)}\right)$ and $s_{2} \in \mathcal{T}\left(\Delta^{(0)} \cup \Delta^{(2)}\right)$. We proceed the same way to show the other conditions of (1), reducing incrementally the possible domain of each component of $w$. The conditions (2) and (3) follow then from the above reductions and the colors of the terms.

Lemma 8 There exists a 01-derivation witness for $R_{1}$ iff there exists a solution for PCP.

Proof: For the if direction, assume that the sequence $\left(i_{j}\right)_{0 \leq j \leq k}$ is a solution of PCP, and let $s:=\left(u_{i_{0}} \otimes v_{i_{0}}\right)\left(u_{i_{1}} \otimes v_{i_{1}}\right) \ldots\left(u_{i_{k}} \otimes v_{i_{k}}\right)$ and $t:=u_{i_{0}} u_{i_{1}} \ldots u_{i_{k}}$. By construction of $R_{1}$, and because $t=v_{i_{0}} v_{i_{1}} \ldots v_{i_{k}}$, the tuple $\left(s^{(0)}, s^{(1)}, s^{(2)}, t^{(10)}, t^{(11)}, t^{(12)}, t^{(17)}, t^{(18)}\right)$ is a 01-derivation witness for $R_{1}$.

For the only if direction, let $\left(s_{0}, s_{1}, s_{2}, t_{10}, t_{11}, t_{12}, t_{17}, t_{18}\right)$ be a 01-derivation witness for $R_{1}$. By (1) and (2) of Lemma 7 , there exist a sequence $\left(i_{j}\right)_{0 \leq j \leq k}$ such that, for each $\ell=0,1,2: s_{\ell}=\left(u_{i_{0}}^{(\ell)} \otimes v_{i_{0}}^{(\ell)}\right) \cdots\left(u_{i_{k}}^{(\ell)} \otimes v_{i_{k}}^{(\ell)}\right)$. The other conditions in (1)-(3) in Lemma 7 imply that $\left(i_{j}\right)_{0 \leq j \leq k}$ is a solution of PCP (see the comments in Figure 1).

Lemmas 6, 7 and 8 establish a reduction of the undecidable PCP into the reachability problem for $\left(R_{1}, 0,1\right)$. Hence we can conclude with the following theorem. 
Theorem 9 The ground reachability problem is undecidable for flat TRS.

\section{Reduction of PCP to joinability for flat TRS}

The undecidability for the joinability follows from a reduction presented in [7] (we can also observe that the joinability problem for $R_{1}, 0$ and 1 is equivalent to the reachability problem for $R_{1}, 0$ and 1 ).

Corollary 10 The ground joinability problem is undecidable for flat TRS.

\section{Reduction of PCP to confluence for flat TRS}

We shall modify the TRS $R_{1}$ in order to reduce PCP to confluence ${ }^{2}$. More precisely, we shall construct a TRS $R_{2}$ such that $0 \underset{R_{1}}{\stackrel{*}{\longrightarrow}} 1$ iff $R_{1} \cup R_{2}$ is confluent.

The TRS $R_{2}$ is defined on the extended signature: $\Xi^{\prime}=\Xi \cup\{2\}$, where 2 has arity 0 in $\Xi^{\prime}$.

$$
\begin{aligned}
R_{2}:= & \{2 \rightarrow 0,2 \rightarrow 1\} \cup\left\{c \rightarrow 0 \mid c \in \Xi_{0} \backslash\{0,1\}\right\} \cup\left\{d(x) \rightarrow 0 \mid d \in \Xi_{1}\right\} \cup \\
& \left\{d(1) \rightarrow 1 \mid d \in \Xi_{1}\right\} \cup\left\{f\left(z_{1}, \ldots, z_{8}\right) \rightarrow 1, g\left(z_{1}, \ldots, z_{8}\right) \rightarrow 1 \mid\right. \\
& \text { one of the } \left.z_{i} \text { is } 1, \text { the others are distinct variables }\right\}
\end{aligned}
$$

We recall that $\Xi_{0}$ and $\Xi_{1}$ denote the set of symbols of $\Xi$ of arity respectively 0 and 1 . Note that $R_{2}$ is flat.

Lemma $11 R_{1} \cup R_{2}$ is confluent iff $0 \stackrel{*}{R_{1}} 1$.

Proof: (sketch) For the only if direction, assume that $0 \stackrel{\digamma_{R_{1}}^{*}}{\rightarrow} 1$. It means that 0 and 1 are not joinable by $R_{1}$ (since 1 is in normal form for $R_{1} \cup R_{2}$ ) and hence also not joinable by $R_{1} \cup R_{2}$. Hence $R_{1} \cup R_{2}$ is not confluent because of the peak $0 \underset{R_{2}}{\overleftarrow{R_{2}}} 2 \underset{R^{\prime}}{\longrightarrow}$.

For the if direction, assume that $0 \stackrel{*}{R_{1}}$, and let $R_{3}:=\left(R_{1} \cup R_{2}\right) \backslash R_{4}$, where $R_{4}$ contains the rules of $T_{A}^{(3)}, T_{A}^{(4)}, T_{A}^{(5)}, T_{A}^{(6)}, T_{B}^{(13)}, T_{B}^{(14)}, T_{B}^{(15)}, T_{B}^{(16)}$ and $0 \rightarrow f\left(q_{A}^{(3)}, q_{A}^{(4)}, q_{A}^{(5)}, q_{B}^{(13)}, q_{B}^{(14)}, q_{A}^{(6)}, q_{B}^{(15)}, q_{B}^{(16)}\right), 2 \rightarrow 0,2 \rightarrow 1$.

We can observe that it is sufficient to show that $R_{3}$ is confluent in order to show that $R_{1} \cup R_{2}$ is confluent. Indeed if $s \underset{R_{1} \cup R_{2}}{\stackrel{*}{R_{1} \cup R_{2}}} t$, and one reduction involves a rule of $R_{4}$, then $u, s$ and $t$ contain at least a constant symbol of $\Xi_{0}$ and then $s \stackrel{*}{R_{2}} 1 \stackrel{*}{R_{2}} t$. Since $R_{3}$ is terminating, we prove its confluence using Newman's lemma, by observing that all its critical pairs can be joined by $R_{2}$.

\footnotetext{
${ }^{2} \mathrm{~A}$ similar technique is used in [6] to show the NP-hardness of confluence for shallow TRS.
} 
By Lemmas 6, 7, 8 and 11, there is a reduction of PCP into the confluence for the flat TRS $R_{1} \cup R_{2}$.

Theorem 12 The confluence is undecidable for flat TRS.

\section{Conclusion}

We have shown that the properties of reachability, joinability and confluence are undecidable for flat (and hence shallow) TRS. This is a big contrast with the shallow linear case, for which all these properties are known to be decidable in polynomial time [6].

One can note that all the known decidability results for confluence concern classes of linear TRS. Two subclasses of (non-linear) shallow TRS remain out of the scope of the reductions constructed here: the shallow rightground TRS - reachability and joinability are decidable for right-ground rewrite systems [9], and, more generally, subclasses of shallow TRS with syntactic restriction on the relative occurrences of variables between left and right members of rules, e.g. shallow TRS such that a variable with more than one occurrence in the left member can not occur in the right member of a rewrite rule.

\section{Acknowledgements}

The author wishes to thank Ralf Treinen who has carefully read and commented a preliminary version of this paper, and Harald Ganzinger for his useful suggestions.

\section{References}

[1] M. Dauchet, T. Heuillard, P. Lescanne, S. Tison, Decidability of the confluence of finite ground term rewrite systems and of other related term rewrite systems, Information and Computation 88 (1990) 187-201.

[2] M. Oyamaguchi, The Church-Rosser property for ground termrewriting systems is decidable, Theoretical Computer Science 49 (1) (1987) 43-79.

[3] A. Hayrapetyan, R. Verma, On the complexity of confluence for ground rewrite systems, in: Bar-Ilan International Symposium on the Foundations of Artificial Intelligence, 2002.

[4] H. Comon, G. Godoy, R. Nieuwenhuis, The confluence of ground term rewrite systems is decidable in polynomial time, in: 42nd Annual IEEE Symposium on Foundations of Computer Science, Las Vegas, Nevada, USA, 2001. 
[5] A. Tiwari, Deciding confluence of certain term rewriting systems in polynomial time, in: G. Plotkin (Ed.), IEEE Symposium on Logic in Computer Science, IEEE Society, 2002, 447-456.

[6] G. Godoy, A. Tiwari, R. Verma, On the confluence of linear shallow term rewrite systems, in: H. Alt (Ed.), 20th Intl. Symposium on Theoretical Aspects of Computer Science, Lecture Notes in Computer Science, Springer, 2003, to appear.

[7] R. Verma, M. Rusinowitch, D. Lugiez, Algorithms and reductions for rewriting problems, Fundamenta Informaticae 43 (3) (2001) 257-276, also in Proc. of Intl Conference on Rewriting Techniques and Applications 1998.

[8] H. Ganzinger, F. Jacquemard, M. Veanes, Rigid reachability, in: Proc. Asian Computing Science Conference, Vol. 1538 of Lecture Notes in Computer Science, Springer-Verlag, Berlin, 1998.

[9] M. Oyamaguchi, The reachability and joinability problems for rightground term-rewriting systems, Journal of Information Processing 13 (3) (1990) 347-354. 